\title{
Nested identification of subjective probabilities
}

\author{
Jacques H. Dreze
}

Received: 21 October 2010 / Accepted: 17 February 2011 / Published online: 25 March 2011

(C) The Author(s) 2011. This article is published with open access at SpringerLink.com

\begin{abstract}
The theory of games against nature relies on complete preferences among all conceivable acts (case 1). Aumann and Drèze (Am Econ J Microecon 1(1):1-16, 2009) consider situations where preferences are defined only for a given set of acts (case 2). We extend these results to situations where (i) only the set of optimal elements from a given set of acts is known (case 3); (ii) only a single optimal act is known (case 4). To these four cases correspond four nested sets of admissible subjective probabilities. Cases 3 and 4 define the extent to which probabilities must be specified to solve a decision problem.
\end{abstract}

Keywords Games $\cdot$ Decisions $\cdot$ Probability $\cdot$ Elicitation

JEL Classification $\quad$ D $81 \cdot$ C 72

\section{Introduction}

The standard model of decision theory, as used e.g. by Savage (1954) or Anscombe and Aumann (1963), proceeds from complete preferences on a comprehensive set of acts. Specifically, let $S$ be the set of states of nature $s$ and $C$ be the set of pure consequences $c$. In Savage, the set of acts $F$ is the set of mappings $f$ of $S$ into $C$. In

\footnotetext{
This paper is an outgrowth of joint research with R.J. Aumann (2009), and I regard it as joint work. Because I wrote up the paper on my own (after some e-mail exchanges), Aumann tactfully declined to appear as co-author. I had to agree, reluctantly, and I thank him warmly for the stimulating cooperation. I have also benefitted from helpful discussions with Jean-François Mertens and Edi Karni. I assume sole responsibility for the contents.
}

J. H. Dreze $(\bowtie)$

CORE, Université catholique de Louvain, Louvain-la-Neuve, Belgium

e-mail: jacques.dreze@uclouvain.be 
Anscombe-Aumann, it is the set of probability distributions over $F$, say $\Delta(F)$. Yet, a standard decision problem calls for the choice of some element from a proper subset of $F$, say the set $R$ of alternatives really open to choice. ${ }^{1}$ Additional acts, elements of $F \backslash R$ or $\Delta(F) \backslash \Delta(R)$, are introduced for analytical convenience, and for the strength of conclusions thereby reached: preferences are represented by subjectively expected utility, with utility $u$ defined uniquely up to positive linear transformations and subjective probability $p$ defined uniquely.

The Savage framework is an extension of that introduced in von Neumann and Morgenstern (1944) where "states" boil down to outcomes of random devices with known (objective) probabilities. In that framework, preferences over "lotteries" are assumed complete, continuous and transitive. But already in von Neumann and Morgenstern (1944, p.19), and next in Aumann (1962), reference is made to the possibility of replacing the completeness axiom by a partial preference ordering over lotteries. The more structured result in that vein is the "extended multi-utility theorem" in Dubra et al. (2004): "there exists a uniquely defined, closed and convex set of utility functions verifying the expected utility theorem".

More recently, Aumann and Drèze (2009) - hereafter ASR — have presented a parallel analysis for decisions in games of strategy (GoS). They look at a game from the view-point of a single player, called "the protagonist"; all other players are combined into a single "opponent". Let then $S$ be the set of the opponent's strategies $s, R$ be the set of the protagonist's strategies $r$, and $C$ be the set of possible outcomes of the game for the protagonist. Each strategy $r \in R$ defines a mapping $h_{r}$ of $S$ into $C$. ASR proceeds from complete preferences over $\Delta(R \cup C)$ and derives a subjective-expected-utility representation of these preferences. Utility is still unique up to positive linear transformations. Subjective probability is in general not unique: there may exist several probabilities, like $p$ and $p^{\prime}$, such that the expected utilities $u_{p}(r)=\Sigma_{s} p_{s} u\left(h_{r}(s)\right)$ and $u_{p^{\prime}}(r)=\Sigma_{s} p_{s}^{\prime} u\left(h_{r}(s)\right)$ are equal, for each $r \in R$ - a property labeled "payoff-equivalence" in ASR. In such cases, preferences over $\Delta(R \cup C)$ do not permit discrimination between $p$ and $p^{\prime}$. And such cases arise when the matrix $\left[u\left(h_{r}(s)\right], r \in R, s \in S\right.$, has rank less than $S$-a situation avoided under a comprehensive set of acts. ${ }^{2}$

The reason for entertaining preferences over $\Delta(R \cup C)$, neither more nor less, is twofold: (i) introducing hypothetical strategies $\tilde{r} \notin R$ changes the game, with potential consequences for preferences and their expected-utility representation; (ii) $R$ will typically fail to include constant strategies, with $h_{r}(s)=c \forall s \in S$, some $c \in C$; accordingly, $u(c)$ cannot be inferred from preferences over $\Delta(R)$ alone; elements of $\Delta(R \cup C)$, called "hybrid lotteries" in ASR, are introduced to that end. The complete preferences over $\Delta(C)$ thus entail a unique (up to positive linear transformations) utility function for consequences.

The main theorem in ASR, hereafter MTASR, which asserts existence of a subjective-expected-utility representation of preferences over $\Delta(R \cup C)$ verifying

\footnotetext{
${ }^{1}$ If I must choose one of two acts, each of which assigns identical consequences to states $s$ and $s^{\prime}$, the respective probabilities of $s$ and $s^{\prime}$ are irrelevant—only their sum matters.

$2 F$ includes acts that "stake a prize" on a single state, and this feature applies to every $s$ in $S$.
} 
payoff-equivalence, is of course applicable to games against nature $(\mathrm{GaN})$ as wellalthough the motivation for restricting attention there to preferences over $\Delta(R \cup C)$ instead of the full $\Delta(F)$ is less compelling. ${ }^{3}$ Still, it is a useful result in that context, because the decision maker might have difficulty in forming meaningful preferences between highly hypothetical options or might be reluctant to evaluate carefully acts that are clearly irrelevant. ${ }^{4}$

In games of strategy, preferences over mixed strategies are meaningful: these are precisely the objects of choice open to the protagonist. Yet, these preferences are not "observable", in particular not subject to (potentially) observable binary choices. The only observable choices concern optimal strategies: the subset, say $M \subset R$, or $\Delta(M) \subset \Delta(R)$, some element of which the protagonist will actually play. Indeed, the protagonist must choose some mixed strategy, and the set of preferred choices is $\Delta(M)$. Thus the definition of $M$, and preferences over $\Delta(M \cup C)$, are "operational" concepts. In fact, they are the very concepts entertained, for a different context, in the "revealed-preferences" theory of Samuelson (1938) and his followers.

Under a more restrictive notion of "operationalism", one might regard a single element of $M$ as "observable", namely the strategy, say $r^{*}$, actually played by the protagonist.

The present paper develops this revealed-preferences approach to decision theory for both GaN and GoS (Sect. 2), and relates it to standard decision theory as well as to ASR (Sect. 3). Our basic result, Theorem 1, is based on axiomatisation of $M$ and of preferences that are complete only on $\Delta(M \cup C)$ : "there exists a uniquely defined convex set of probabilities on $S$ verifying (together with $u(c)$ ) the subjective expected utility theorem".

The parallelism with the main theorem in Dubra et al. (2004) is striking. Starting from known probabilities, these authors elicit from incomplete preferences over lotteries a set of admissible utility functions. Starting instead from a fully elicited utility function, we derive from incomplete preferences over acts a set of admissible probabilities.

Corollary 2 treats the case where preferences are complete only on $\Delta\left(r^{*} \cup C\right)$. Corollary 3 in Sect. 3 offers a concise summary conclusion by relating the extent of identification of subjective probabilities to the number of linearly independent acts or strategies over which preferences are observed.

For completeness, Sect. 4 relates our results to those obtaining in the related but distinct context of "games of strength and skill" (in the terminology of von Neumann and Morgenstern (1944)) or "games with moral hazard" (in the terminology of Drèze (1987)); namely one-person games where the occurence of the "states" is influenced by the (unobserved) strategy choices of the decision maker. In that context, alternative probabilities are associated with alternative strategy choices, but a parallel identification issue arises.

Some concluding remarks are offered in Sect. 5.

\footnotetext{
3 In particular, adding hypothetical acts does not affect nature's choices.

${ }^{4}$ In particular, existence of a dominant strategy eliminates the need to assess alternatives.
} 


\section{Basic result}

\section{1}

We adopt the notation of ASR, and interpret it indifferently for GaN's or for GoS's. A game $G$ consists of

- a finite set $R$ with elements $r$ (the pure strategies of the protagonist or the acts of the decision maker),

- a finite set $S$ with elements $s$ (the pure strategies of the opponent or the states of nature; states for short),

- a finite set $C$ with elements $c$ (pure consequences),

- a function $h: R \times S \rightarrow C$ (the outcome function of the protagonist in a GoS or the definition of the acts in a $\mathrm{GaN})$.

Thus, $G=(R, S, C, h)$. We write $h_{r}(s)$ for the consequence associated with the pair $(r, s) \in R \times S$.

For a finite set $A$, the set of probability distributions on $A$ is denoted $\Delta(A)$, with elements $\alpha$. Thus, $\gamma \in \Delta(C)$ is a mixed consequence, and $\rho \in \Delta(R)$ is a mixed strategy in a GoS or a lottery over acts in a GaN. We also write $\rho_{s}$ for the mixed consequence associated by $\rho$ with state $s$. As for $\Delta(R \cup C)$, with elements $\lambda$, it is a set of hybrid lotteries defined by triplets $\left(\rho^{\lambda}, \gamma^{\lambda}, t^{\lambda}\right) \in \Delta(R) \times \Delta(C) \times[0,1]$. In state $s$, the hybrid lottery $\lambda$ entails the mixed consequence $\lambda_{s}$ yielding $\rho_{s}^{\lambda}$ with probability $t^{\lambda}$ and $\gamma^{\lambda}$ with probability $\left(1-t^{\lambda}\right)$; so, we write $\lambda_{s}=t^{\lambda} \rho_{s}^{\lambda}+\left(1-t^{\lambda}\right) \gamma^{\lambda} \in \Delta(C)$. Similarly, when $\varrho$ is a lottery between $r$ and $r^{\prime}$ with probabilities $(t, 1-t)$, we write $\varrho=t r+(1-t) r^{\prime}$ or $\varrho=\left(r, r^{\prime} ; t\right)$.

In order to develop our "revealed preference" analysis, we start from a partial ordering $\succsim$ on $\Delta(R \cup C)$, which in particular separates a set of preferred mixed strategies $\Delta(M), M \subseteq R$, from the remaining mixed strategies, $\Delta(R) \backslash \Delta(M)$. The interpretation is that the protagonist in the game $G$ is indifferent between playing any strategy $\rho \in \Delta(M)$ but will not play any $\rho^{\prime} \in \Delta(R) \backslash \Delta(M)$.

Three assumptions characterize our partial ordering $\succsim$ on $\Delta(R \cup C)$. By definition, $\succsim$ is transitive and reflexive; but it is not necessarily complete; it embodies the usual definitions of indifference $(\sim)$ and strict preference $(\succ)$.

Assumption 1 There exists $M \subseteq R, M \neq \emptyset$, such that: $\rho \sim \rho^{\prime} \forall \rho, \rho^{\prime} \in \Delta(M)$ and $\rho \succ \rho^{\prime} \forall \rho \in \Delta(M), \rho^{\prime} \in \Delta(R) \backslash \Delta(M)$.

Assumption 1 amounts to the assertion that the protagonist will play the game $(M \neq \emptyset)$, and reveals her full set of preferred strategies $\Delta(M)$.

Next, we define a complete preference ordering $\succsim$ on a set $\Delta(A)$ to be an $N-M$ preference ordering if it satisfies the standard axioms of utility theory ${ }^{5}$, as stated for instance in von Neumann and Morgenstern (1944) or Luce and Raiffa (1957). And we define an $N-M$ utility on $\Delta(A)$ to be a real-valued function $u$ on $\Delta(A)$ such that, $\forall \alpha, \alpha^{\prime} \in \Delta(A)$ and $\forall t \in[0,1]$,

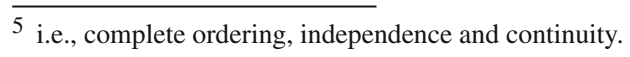


- $\alpha \succsim \alpha^{\prime}$ iff $u(\alpha) \geq u\left(\alpha^{\prime}\right)$

- $u\left(t \alpha+(1-t) \alpha^{\prime}\right)=t u(\alpha)+(1-t) u\left(\alpha^{\prime}\right)$.

As is well known, an $N-M$ preference admits an $N-M$ utility representation.

Assumption 2 The restriction of $\succsim$ to $\Delta(M \cup C)$ is an $N-M$ preference.

Thus, on $\Delta(M \cup C)$, the preference ordering is complete and admits an $N-M$ utility representation.

Assumption 3 For $\lambda, \lambda^{\prime} \in \Delta(R \cup C)$, if $\lambda_{s} \succsim(\succ) \lambda_{s}^{\prime} \forall s \in S$, then $\lambda \succsim(\succ) \lambda^{\prime}$.

Assumption 3 introduces a condition of monotonicity which extends our partial preference ordering to those elements of $\Delta(R \cup C) \backslash \Delta(M \cup C)$ among which a (weak) preference domination holds. Note that $\lambda_{s} \succsim \lambda_{s}^{\prime}$ is well defined in view of Assumption 2, applied to $\Delta(C) \subset \Delta(M \cup C)$. Assumption 3 embodies the "reversal of order" condition of Anscombe and Aumann (1963) and a weak form of the "sure-thing principle" of Savage (1954). ${ }^{6}$

Assumptions 1-3 have an important implication.

Proposition 1 Let $\rho, \rho^{\prime}, \rho^{\prime \prime} \in \Delta(R)$ be such that, for some $t \in(0,1), \rho_{s} \sim t \rho_{s}^{\prime}+$ $(1-t) \rho_{s}^{\prime \prime}$ for all $s \in S$; then, $\rho \in \Delta(M)$ if and only if $\rho^{\prime} \in \Delta(M)$ and $\rho^{\prime \prime} \in \Delta(M)$.

Proof By Assumption 3, $\rho$ is indifferent to $t \rho^{\prime}+(1-t) \rho^{\prime \prime}$. If $\rho \in \Delta(M)$, then $t \rho^{\prime}+(1-t) \rho^{\prime \prime} \in \Delta(M)$, with $\varrho \succsim \varrho^{\prime}$ and $\varrho \succsim \varrho^{\prime \prime}$. Accordingly, by Assumption 2, $\rho \sim \rho^{\prime}$ and $\rho \sim \rho^{\prime \prime}$, so that $\rho^{\prime} \in \Delta(M)$ and $\rho^{\prime \prime} \in \Delta(M)$. Conversely, either $\rho^{\prime} \notin \Delta(M)$ or $\rho^{\prime \prime} \notin \Delta(M)$ implies $\rho \notin \Delta(M)$.

This is a natural property: in GaN, $\rho$ could not be part of the preferred set $\Delta(M)$ if it is a convex combination (preference wise) of a preferred and a discarded strategy, or of two discarded strategies. The status of this property in GoS is discussed in Sect. 5.2.

Theorem 1 Under Assumptions 1, 2 and 3, there exist:

- an $N-M$ utility $u$ on $\Delta(C)$,

- a non-empty convex set $\Gamma \subset \Delta(C)$ such that $\gamma \in \Gamma$ and $\rho \in \Delta(M)$ imply $\gamma \sim \rho$,

- a non-empty convex set $P_{3}$ of probabilities on $S$ such that, for all $p \in P_{3}$, but only for $p \in P_{3}$ :

(i) $u_{p}(\rho):=\Sigma_{s \in S} p_{s} u\left(\rho_{s}\right)=u(\gamma) \forall \rho \in \Delta(M), \gamma \in \Gamma$;

(ii) $u_{p}(\rho)>u_{p}\left(\rho^{\prime}\right) \forall \rho \in \Delta(M), \rho^{\prime} \in \Delta(R) \backslash \Delta(M)$.

This theorem establishes that the choice by the protagonist of the set $M$ of preferred strategies is sustained by a subjective expected utility analysis, where probabilities are in general not unique, but satisfy payoff equivalence over $\Delta(M)$. Indeed, (i) implies $u(\gamma)=u_{p}(\rho)=u_{p^{\prime}}(\rho)$ for all $p, p^{\prime} \in P_{3}$ and $\rho \in \Delta(M) .{ }^{7}$ Theorem 1 covers case 3

\footnotetext{
6 "Reversal of order" is defined and discussed in Sect. 4.

7 It is not claimed that $u_{p}(\varrho)=u_{p^{\prime}}(\varrho)$ when $\varrho \in \Delta(R) \backslash \Delta(M)$. And it is not claimed that $P_{3}$ is closed. For instance, let $S=\{1,2\}, r=\left(\frac{1}{2}, \frac{1}{2}\right) \succ r^{\prime}=\left(\frac{3}{4}, \frac{1}{4}\right)$ and $R=\left\{r, r^{\prime}\right\}$. Disregarding $r^{\prime}, P_{3}$ contains every probability $p=(t, 1-t), t \in[0,1]$. Taking $r^{\prime}$ into account imposes $t<\frac{1}{2}$.
} 
in the abstract (hence the notation $P_{3}$ ). Because $P_{3}$ is convex, it can be defined as containing all the vectors $p$ verifying (i) and (ii); in that sense, $P_{3}$ is unique.

Note that conclusion (i) of the theorem is implied by MTSAR applied under restriction of $R$ to $M$. Under that restriction, $\Delta(R) \backslash \Delta(M)$ is an empty set, and conclusion (ii) is void. The proof to follow is needed to obtain (ii), then Corollary 1 . Note further that, when $\Delta(R) \backslash \Delta(M) \neq \emptyset$, the elements of that set matter to the contents of $P_{3}$, though not to its dimension (number of linearly independent elements). In contrast, the elicitation of $\Gamma$ may reduce by one the dimension of $P_{3}$.

The logic of the proof is elementary. Represent an act or strategy $r$ in $R$ by the $S$-vector of its (expected) utilities. $\Delta(R)$ is then described by a convex polyhedron in $S$-space, and $\Delta(M)$ is a face of that polyhedron. $P_{3}$ is the set of vectors normal to that face, hence has complementary dimension. ${ }^{8}$

Corollary 1 If $\Delta(M \cup \Gamma)$ contains $k$, and at most $k$, linearly independent elements, then $P_{3}$ contains $S-k+1$, and at most $S-k+1$ linearly independent elements.

Proof $P_{3}$ is the set of solutions to the system of linear equalities and inequalities

$$
u_{p}(\varrho)=\tilde{\gamma}, \varrho \in \Delta(M), u_{p}(\varrho)<\tilde{\gamma}, \varrho \in \Delta(R) \backslash \Delta(M), \tilde{\gamma} \in \Gamma
$$

There are $k$ (and at most $k$ ) linearly independent equalities in $S$-space. Corollary 1 is equivalent to Proposition 2.4 , p. 87 of Nemhauser and Wolsey (1988). ${ }^{9}$

\section{2}

Proof of Theorem 1 Assume w.l.o.g. that $C$ contains $\gamma, \gamma^{\prime}$ with $\gamma \succ \gamma^{\prime}$. (Otherwise, take $u$ to be identically 0 , and $P_{3}$ the set of all probability distributions on $S$.)

From Assumption 2, we obtain directly the utility $u$, which we normalize (arbitrarily), and the set $\Gamma$. Next, we eliminate temporarily from consideration any state $\hat{s} \in S$ such that there exist $\rho, \rho^{\prime} \in \Delta(M)$ with $\rho_{s} \sim \rho_{s}^{\prime} \forall s$ and $\rho_{\hat{s}} \succ \rho_{\hat{s}}^{\prime}$. Indeed, it will be the case that $p_{\hat{s}}=0 \forall p \in P_{3}$. Denote by $\bar{S}$ the set of remaining states, i.e. those not thereby eliminated.

Convention: for the rest of this proof, we represent a hybrid lottery $\lambda$ by the $\bar{S}$-vector of the (expected) utilities $u\left(\lambda_{s}\right)$ of its mixed consequences in states $s \in \bar{S}$. Thus, $\lambda$ is a point in Euclidean $\bar{S}$-space $\Re^{\bar{S}}$.

Preference relations among consequences are thus replaced by inequalities among utilities; every hybrid lottery in $\Delta(R \cup C)$ is defined by a point in $\Re^{\bar{S}}$; and every $\gamma \in \Delta(C)$ is a point on the main diagonal of $\Re^{\bar{S}}$. We henceforth write $\tilde{\gamma}$ for the unique

\footnotetext{
$\overline{8}$ In the example of footnote $7, M=\{r\}$ contains a single element, and $P_{3}=\left\{t \in[0,1]: t<\frac{1}{2}\right\}$ contains $S=2$ linearly independent elements.

9 Alternatively stated, $P_{3}$ defines the null space of the $S \times k$ matrix $U-V_{\gamma}$, where columns of $U$ are linearly independent utility vectors associated with elements of $\Delta(M \cup \Gamma)$ and $V_{\gamma}$ is the $S \times k$ matrix with all elements equal to $\bar{\gamma}$-so that $p\left(U-V_{\gamma}\right)=0$ for all $p$ in $P_{3}$. The rank of $U-V_{\gamma}$ is $k-1$, since $\gamma \in M \cup \Gamma$.
} 
point of the main diagonal of $\Re^{\bar{S}}$ corresponding to $\Gamma$. As for $\Delta(R)$, it is a convex compact polyhedron in $\Re^{\bar{S}}$, with extreme points in $R$.

By the definition of $\bar{S}$, if $\rho \in \Delta(M)$, then there does not exist $\rho^{\prime} \in \Delta(R)$ with $\rho^{\prime}>\rho .{ }^{10}$ Accordingly, for every $\rho \in \Delta(M),\left\{\rho+\Re_{+}^{\bar{S}}\right\} \cap \Delta(R)=\{\rho\}$.

We now consider the sets of hybrid lotteries $\Delta(M \cup \Gamma)$ and $\Delta(R \cup \Gamma) \in \Re^{\bar{S}}$. These subsets of $\Delta(M \cup C)$ and $\Delta(R \cup C)$ respectively correspond to the convex hulls of the union of $M$, respectively $R$, with the point $\tilde{\gamma}$ on the main diagonal of $\Re^{\bar{S}}$ defining the utility of a mixed consequence indifferent to playing the game $G$. (That is, $\tilde{\gamma}$ is the subjective value of $G$.)

The properties of $\Delta(M) \subseteq \Delta(R)$ also hold for $\Delta(M \cup \Gamma) \subseteq \Delta(R \cup \Gamma)$, in particular Proposition 1.

Lemma 1 Let $\lambda, \lambda^{\prime}, \lambda^{\prime \prime} \in \Delta(R \cup \Gamma)$ be such that, for some $\tau \in(0,1), \lambda_{s}=\tau \lambda_{s}^{\prime}+$ $(1-\tau) \lambda_{s}^{\prime \prime} \forall s \in \bar{S}$; then, $\lambda \in \Delta(M \cup \Gamma)$ if and only if $\lambda^{\prime} \in \Delta(M \cup \Gamma)$ and $\lambda^{\prime \prime} \in \Delta(M \cup \Gamma)$.

Proof With $\lambda:=t \rho+(1-t) \tilde{\gamma}, \lambda^{\prime}:=t^{\prime} \rho^{\prime}+\left(1-t^{\prime}\right) \tilde{\gamma}, \lambda^{\prime \prime}:=t^{\prime \prime} \varrho^{\prime \prime}+\left(1-t^{\prime \prime}\right) \tilde{\gamma}$ and $\lambda=\tau \lambda^{\prime}+(1-\tau) \lambda^{\prime \prime}$, let $\hat{\tau}:=\tau t^{\prime}+(1-\tau) t^{\prime \prime} \in(0,1)$ and $\hat{\rho}=\frac{\tau t^{\prime} \rho^{\prime}+(1-\tau) t^{\prime \prime} \rho^{\prime \prime}}{\hat{\tau}}$, so that $\lambda=\hat{\tau} \hat{\rho}+(1-\hat{\tau}) \tilde{\gamma}$. If $\lambda \in \Delta(M \cup \Gamma)$ so that $\lambda \sim \rho \sim \tilde{\gamma}$, then $\lambda \sim$ $\hat{\tau} \rho+(1-\hat{\tau}) \gamma \sim \hat{\tau} \hat{\rho}+(1-\hat{\tau}) \tilde{\gamma}$ implying $\rho \sim \hat{\rho}$. By Proposition $1, \rho \in \Delta(M)$ if and only if $\rho^{\prime}, \rho^{\prime \prime} \in \Delta(M)$ hence $\lambda^{\prime}, \lambda^{\prime \prime} \in \Delta(M \cup \Gamma)$.

It follows from lemma 1 that, for every $\lambda \in \Delta(M \cup \Gamma),\left\{\lambda+\Re_{+}^{\bar{S}}\right\} \cap \Delta(R \cup \Gamma)=\{\lambda\}$. It also follows that $\Delta(M \cup \Gamma)$ is a face of the polyhedron $\Delta(R \cup \Gamma){ }^{11}$

Lemma 2 Either $\Delta(M)=\Delta(R)$ or $\Delta(M \cup \Gamma) \cap$ ri $\Delta(R \cup \Gamma)=\emptyset$, where ri stands for "relative interior".

Proof See Corollary 18.1.3 in Rockafellar (1970).

To prove Theorem 1, let then $M^{*}:=\cup_{\lambda \in \Delta(M \cup \Gamma)}\left\{\lambda+\Re_{+}^{\bar{S}}\right\}$, a convex set. By lemma 2 , ri $M^{*} \cap$ ri $\Delta(R \cup \Gamma)=\emptyset$. Accordingly there exists a hyperplane, say B separating $M^{*}$ from $\Delta(R \cup \Gamma)$ (Rockafellar 1970, Theorem 11.3), and containing $\Delta(M \cup \Gamma)=$ $M^{*} \cap \Delta(R \cup \Gamma)$. And there exists a normal vector to $\mathrm{B}$, say $\bar{p}$, with $\bar{p} \gg 0, \Sigma_{s \in \bar{S}} \bar{p}_{s}=$ $1, \bar{p} \lambda=\bar{p} \lambda^{\prime} \forall \lambda, \lambda^{\prime} \in \Delta(M \cup \Gamma)$ and $\bar{p} \lambda>\bar{p} \lambda^{\prime} \forall \lambda \in \Delta(M \cup \Gamma), \lambda^{\prime} \in \Delta(R \cup \Gamma) \backslash$ $\Delta(M \cup \Gamma)$.

Denote by $\bar{P}_{3}$ the set of all vectors in $\Re_{+}^{\bar{S}}$ verifying these four properties. We have just shown that $\bar{P}_{3}$ is non-empty. Also, $\bar{P}_{3}$ is convex because each of the four defining properties is preserved under convex combinations. ${ }^{12}$

Abandoning our convention, let $P_{3} \subset \Re^{S}$ be the set of vectors $p$ defined by: $\exists \bar{p} \in \bar{P}_{3}, p_{\bar{S}}=\bar{p} ; p_{s}=0 \forall s \in S \backslash \bar{S}$. That is, the restriction of $P_{3}$ to $\Re^{\bar{S}}$ is given by $\bar{P}_{3}$ and the restriction of $P_{3}$ to $\Re S \backslash \bar{S}$ is the zero vector. Then $P_{3}$ satisfies conclusions (i) and (ii) of Theorem 1.

\footnotetext{
$\overline{10}$ Vector inequalities are $\geq,>, \gg$.

11 "A face of a convex set $C$ is a convex subset $C^{\prime}$ of $C$ such that every (closed) line segment in $C$ with a relative interior point in $C^{\prime}$ has both endpoints in $C^{\prime}$ " (Rockafellar 1970, p. 162).

12 Actually $\bar{P}_{3}$ is (i) the union of the normal cones to the set of hyperplanes containing $\Delta(M)$ and separating $\Delta(R)$ from $\left\{x+\mathfrak{R}_{+}^{\bar{S}}, x \in \Delta(M)\right\}$, (ii) intersected with the unit simplex of $\Re_{+}^{\bar{S}}$.
} 


\section{Nested identification of subjective probabilities}

\section{1}

To cover case 4 in the abstract, we now introduce the corollary to Theorem 1 holding when Assumptions 1 and 2 are weakened as follows:

Assumption 1* There exists $r^{*} \in R$ such that $r^{*} \succsim \rho \forall \rho \in \Delta(R)$.

Assumption $2^{*}$ The restriction of $\succsim$ to $\Delta\left(r^{*} \cup C\right)$ is an $N-M$ preference.

Corollary 2 Under Assumptions $1 *, 2 *$ and 3 there exist:

- an $N-M$ utility u on $\Delta(C)$,

- a non-empty convex set $\Gamma \subset \Delta(C)$ such that $\gamma \in \Gamma$ implies $\gamma \sim r^{*}$,

- a non-empty convex set $P_{4}$ of probabilities on $S, P_{4} \supseteq P_{3}$, such that, for all $p \in P_{4}$, but only for $p \in P_{4}$ :

(i) $u_{p}\left(r^{*}\right)=\Sigma_{s \in S} p_{s} u\left(r_{s}^{*}\right)=u(\gamma) \forall \gamma \in \Gamma$;

(ii) $u_{p}\left(r^{*}\right) \geq u_{p}(\rho) \forall \rho \in \Delta(R)$.

Proof Repeating step by step the reasoning in the proof of Theorem 1, with $M$ systematically replaced by $\left\{r^{*}\right\}$, we obtain successively the $N-M$ utility $u$ on $\Delta(C)$, the set $\Gamma$ and a non-empty convex set of probabilities $P_{4}$ satisfying conclusions (i) and (ii) of Corollary 2. Furthermore, $P_{4} \supseteq P_{3}$ because every $p \in P_{3}$ satisfies conclusions (i) and (ii) in Corollary 2, and $P_{4}$ is comprehensive.

Remark When $r^{*}$ in $\Gamma$ is a strictly dominant strategy, $P_{4}$ is the unit simplex of $\Re^{S}$ (and $\bar{S}^{*}=S$ ). When $r^{*}$ belongs to the relative interior of $\Delta(M), P_{4}=P_{3} .{ }^{13}$

\section{2}

Turning to case 2 in the abstract, ASR rests on Assumption 3 and

Assumption 2', There is an $N-M$ preference $\succsim$ on $\Delta(R \cup C)$.

Theorem 2 Under Assumptions 2' and 3, there exist

- an $N$-M utility on $\Delta(C)$,

- a non-empty convex set $P_{2} \subseteq P_{3}$ such that, for all $\lambda, \lambda^{\prime} \in \Delta(R \cup C), \lambda \succsim \lambda^{\prime}$ iff, for each $p \in P_{2}$, but only for $p \in P_{2}, u_{p}(\lambda) \geq u_{p}\left(\lambda^{\prime}\right)$,

- for all $\lambda \in \Delta(R \cup C)$, for all $p, p^{\prime} \in P_{2}, u_{p}(\lambda)=u_{p^{\prime}}(\lambda)$.

\footnotetext{
13 If $r^{*}$ is a pure strategy (or act), then it belongs to $r \Delta(M)$ only if $M=r^{*}$; if $r^{*}$ is allowed to be a mixed strategy, the remark assumes significance.
} 
Proof of Theorem $2{ }^{14}$ By Assumption 2', the preferences $\succsim$ on $\Delta(R \cup C)$ admit a representation through an $N-M$ utility $V$ such that, for all $\lambda$ in $\Delta(R \cup C)$

$$
V(\lambda)=\sum_{r \in R} t^{\lambda}(r) V(r)+\sum_{c \in C} t^{\lambda}(c) V(c)
$$

For $\varrho \in \Delta(M)$, for every $p \in P_{3}$,

$$
V(\varrho)=\sum_{s} p_{s} u\left(h_{\varrho}(s)\right)=\bar{\gamma}
$$

Let $\underline{r} \in R$ be such that $r \succsim \underline{r}$ for all $r \in R$; and let $r^{*}$ belong to $M$, so that $r^{*} \succsim r$ for all $r \in R$. Define $\bar{\varrho}=\frac{1}{2} \underline{r}+\frac{1}{2} r^{*}$. (The mean is convenient, but any other intermediate level would do.) Let then $I \subset \Delta(R \cup C)$ denote the indifference class

$$
I:=\{\lambda \in \Delta(R \cup C): \lambda \sim \bar{\varrho}\} .
$$

and let $\Delta_{I}:=\{\lambda \in \Delta(R \cup C): \lambda \precsim \bar{\varrho}\}$ denote the set of lotteries in $\Delta(R \cup C)$ with expected utility not exceeding $\bar{\rho}$. Thus, $I$ is the preferred set for $\Delta_{I}$ :

$$
\forall \varrho, \varrho^{\prime} \in I, \varrho \sim \varrho^{\prime} ; \forall \varrho \in I, \varrho^{\prime} \in \Delta_{I} \backslash I, \varrho \succ \varrho^{\prime}
$$

We may apply Theorem 1 with $\left(I, \Delta_{I}\right)$ taking the place of $(M, R)$, thus obtaining a convex set of probabilities $P_{I}$ with the properties of $P_{3}$ in Theorem 1. This set $P_{I}$ is the set $P_{2}$ of Theorem 2 .

Indeed, $V(\bar{\varrho})=\frac{1}{2} V(\underline{r})+\frac{1}{2} V\left(r^{*}\right)=\frac{1}{2} V(\underline{r})+\frac{1}{2} \tilde{\gamma}$. Hence, for all $p \in P_{I}$,

$$
V(\bar{\varrho})=\frac{1}{2} u_{p}(\underline{r})+\frac{1}{2} u_{p}\left(r^{*}\right)=\frac{1}{2} u_{p}(\underline{r})+\frac{1}{2} \tilde{\gamma},
$$

implying $u_{p}\left(r^{*}\right)=u_{p^{\prime}}\left(r^{*}\right)=\tilde{\gamma}$ and $u_{p}(\underline{r})=u_{p^{\prime}}(\underline{r})$ for all $p, p^{\prime}$ in $P_{I}$. This last property is readily extended to every $r$ in $R$ as follows. If $V(r)>\bar{\varrho}$, there exists $t$ in $(0,1)$ such that $t r+(1-t) \underline{r} \in I$; if $V(r)<\bar{\varrho}$, there exists $t$ in $(0,1)$ such that $t r+(1-t) r^{*} \in I$. In either case, it follows that $u_{p}(r)=u_{p^{\prime}}(r)=V(r)$ for all $p, p^{\prime}$ in $P_{I}=P_{2}$. And $P_{2} \subseteq P_{3}$ because $P_{3}$ is comprehensive by definition.

Corollary 3 (i) If $\Delta(R \cup C)$ contains $k$, and at most $k$ linearly independent elements, then $P_{2}$ contains $S-k+1$, and at most $S-k+1$, linearly independent elements.

(ii) If $R=F$ and $C$ contains $c, c^{\prime}$ with $c \succ c^{\prime}$, then $P_{2}$ is a singleton.

Proof (i) follows from Corollary 1, and (ii) follows from (i).

\footnotetext{
14 Theorem 2 could be proved by invoking MTASR, or lemma 1 in Castagnoli et al. (2003). We offer a proof invoking Theorem 1, thereby paving the way for Corollary 3 and making this paper self-contained.
} 
In a sense, Corollary 3 offers a concise interpretation of this paper: if subjective probabilities are elicited from complete preferences among $k$ acts (or strategies) with linearly independent vectors of state-contingent utilities, the set admissible probabilities $P_{k}$ is $(S-k+1)$-dimensional. If one adds another linearly independent act, $P_{k+1}$ is $(S-k)$-dimensional, with $P_{k+1} \subset P_{k}$. This summarises the "nested identification" feature.

\section{Complement: relation to moral hazard}

As a natural complement, we relate our result on the dimension of $P_{3}$ or $P_{2}$ in Corollaries 1 and 3 to a similar result characterising the set of subjective probabilities in a game with moral hazard (GwMH); see Drèze (1987, Sects. 6 and 7, in particular lemma 7.2). In that context, the decision maker can influence the occurence of the "states" through unobserved strategies. ${ }^{15}$ Strategy choices are guided by the utility of consequences, as per the "Generalised Moral Expectation" Theorem 6.1 in Drèze (1987). In the terminology and notation of the present paper, that theorem reads: ${ }^{16}$

"Under the maintained assumptions, when $R=F$, there exist a closed convex set $P$ of probabilities on $S$, and a utility $u$ on $C$, such that, for all $G$

$$
V(G)=\operatorname{Max}_{p \in P} \sum_{s} p_{s} u\left(h_{G}(s)\right)
$$

$P$ is unique and $u$ is unique up to the same linear transformation as $V . "$

The maintained assumptions include: (i) the $N-M$ assumptions leading to a value function $V$ on games and a utility function $u$ on (mixed) consequences; (ii) a weakening of our monotonicity assumption, explained below: and (iii) a specific assumption (CGO), also explained below.

The gist of GwMH concerns "reversal of order". Let the two games $G:=r$ and $G^{\prime}:=r^{\prime}$ imply different optimal strategies, entailing respectively the unique probabilities $p$ and $p^{\prime}, p \neq p^{\prime}$. Under the lottery $\varrho=\left(r, r^{\prime} ; t\right)$, a random device will select either $r$ or $r^{\prime}$; if $r$, the decision maker selects $p$; if $r^{\prime}$, she selects $p^{\prime}$. The value of $\varrho$ is thus:

$$
V(\varrho)=t \operatorname{Max}_{p \in P} \sum_{s} p_{s} u\left(h_{r}(s)\right)+(1-t) \underset{p^{\prime} \in P}{\operatorname{Max}} \sum_{s} p_{s}^{\prime} u\left(h_{r}(s)\right) .
$$

But there also exists a game $r^{\prime \prime}$ defined by

$$
\begin{aligned}
& h_{r^{\prime \prime}}(s)=t h_{r}(s)+(1-t) h_{r^{\prime}}(s), \\
& V\left(r^{\prime \prime}\right)=\operatorname{Max}_{p^{\prime \prime} \in P} \sum_{s} p_{s}^{\prime \prime}\left[t u\left(h_{r}(s)\right)+(1-t) u\left(h_{r^{\prime}}(s)\right)\right]<V(\varrho)
\end{aligned}
$$

\footnotetext{
15 For instance, commuting by train rather than car reduces the probability of accidental death for a subscriber of life insurance.

16 Note that the result (GME) is used in numerous applications (e.g. to insurance with moral hazard, health economics or safety expenditures) — generically without concern for the underlying axiomatic justification...
} 
where the last inequality follows from $p \neq p^{\prime}$. In contrast, the monotonicity Assumption 3 , imposes $V\left(r^{\prime \prime}\right)=V(\varrho)$, that is "reversal of order". It rules out strict preference for the "immediate lottery" $\rho$ over the corresponding "delayed lottery" $r$ " .17 That is, it rules out a positive "value of information" about the outcome of the lottery. It is thus not a reasonable assumption for GwMH. Accordingly, Drèze (1987) replaces Assumption 3 by the combination of "non-negative value of information"i.e. $V(\varrho) \geq V\left(r^{\prime \prime}\right)$ - and "conditional preferences are well-defined"-the "sure thing principle".

Now, there are also situations where two games $r$ and $r^{\prime}$ are such that $p=p^{\prime}$ : the two games $r$ and $r^{\prime}$ have a common optimal strategy. Drèze (1987) labels such games "equipotent". As a transparent example, let $r$ be "constant", i.e. such that $u\left(h_{r}(s)\right)=u\left(h_{r}\left(s^{\prime}\right)\right)$ for all $s, s^{\prime}$ in $S$; then $V(r)$ is independent of $p$, and $r$ is equipotent with every other game. Such a game is labeled "omnipotent" in Drèze (1987). The assumption CGO states that every constant game is omnipotent.

Of course, there may exist omnipotent games that are not constant. The diversity of consequence profiles among omnipotent games is related to the dimensionality of the set $P$ of attainable probabilities, as per the following lemma (7.2 in Drèze 1987):

"If $P$ contains $S-k+1$, and at most $S-k+1(S \geq k \geq 1)$ linearly independent elements, then the expected utility vectors associated with omnipotent games form a $k$-dimensional subset of $\Re^{S}$."

This last result is formally comparable to Corollaries 1 and 3 above. The relevance of the dimension of $P$ in GwMH is at variance with its relevance in GaN. In the latter case, the extent of identification of subjective probabilities decreases with the dimension of $P$. In the former case, that dimension becomes relevant to handle the more general case of "state-dependent preferences" (treated in Sect. 8 of Drèze 1987); namely the case where consequences cannot be defined without reference to the state that obtains (think again about life insurance, medical insurance or safety expenditures). State-dependent preferences can be represented by state-dependent utilities $u_{s}(c)$, each defined up to a state-specific linear transformation. The extent of identification of the respective origins and units of scale of the state-dependent utilities (which matter to decisions) increases with the dimension of $P$. It stands to reason that the loss of utility associated with an event like death cannot be elicited from insurance decisions-only from decisions affecting the probability of death, through the choice of alternative $p$ 's in $P$. The existence of such alternatives (the dimension of $P$ ) is thus essential for the identification of state-dependent utilities.

Note finally that neither the model in Dubra et al. (2004) nor the model in Sects. 2 and 3 above, is applicable under state-dependent preferences. Indeed, restricting acts to "lotteries" implies state independent preferences. And so does Assumption 2 (or 2* or 2').

17 "Immediate": drawn before observing the state; "delayed": drawn after observing the state. 


\section{Concluding remarks}

\section{1}

In GaN, one can apply the $N-M$ axioms to preferences over four nested sets, namely $\Delta\left(r^{*} \cup C\right), \Delta(M \cup C), \Delta(R \cup C)$ and $\Delta(F) \equiv \Delta(F \cup C)$ - thereby obtaining four nested sets of subjective probabilities, $P_{4}, P_{3}, P_{2}$ and the singleton $\{\tilde{p}\}$. There exist special situations where $P_{4}=P_{3}=P_{2}=\{p\}$. In general, the set inclusions are proper: $P_{4} \supset P_{3} \supset P_{2} \supset\{\tilde{p}\}$.

The standard analysis, based on $\Delta(F)$, brings out the logic of subjective-expectedutility analysis in the most demanding case: when $P_{3}=\{\tilde{p}\}$, precise assessment of probabilities is needed to solve the decision problem. Yet, typical decision situations are less demanding. The sets $P_{4}$ and $P_{3}$ then define the extent to which probabilities need be specified in order to sustain an optimal decision. One measure of that extent is offered by Corollary 3, which relates the dimension of the set of relevant probabilities to the dimension of the decision problem (both measured by numbers of linearly independent elements). But "dimension" is only one aspect of "identification".

By way of illustration, let $S=\{s, t\}$ and $R=\left\{r, r^{\prime}, r^{\prime \prime}\right\}$ where the three acts $r, r^{\prime}, r^{\prime \prime}$ entail the respective utility vectors $\left(\frac{4}{3}, \frac{1}{2}\right),(1,1),\left(\frac{1}{2}, \frac{4}{3}\right)$ in states $(s, t)$. Then $r \succsim r^{\prime}$ iff $p_{s} \geq \frac{3}{5}$ and $r^{\prime} \succsim r^{\prime \prime}$ iff $p_{s} \geq \frac{2}{5}$. The information about $p_{s}$ needed to choose an optimal act amounts to locating $p_{s}$ relative to the interval $\left[\frac{2}{5}, \frac{3}{5}\right]$. This is less demanding than point estimation.

In the same illustration, let $M=\left\{r^{\prime}\right\}=\{(1,1)\}$. Then, $P_{3}=\left(\frac{2}{5}, \frac{3}{5}\right)$. Assume further (Assumption 2') that $r \sim\left(\frac{11}{12}, \frac{11}{12}\right)$. Then $P_{2}=\{\tilde{p}\}=\left\{\frac{1}{2}\right\}$.

Letting instead $M=\{r\}$, and $r \sim\left(\frac{9}{8}, \frac{9}{8}\right)$, then $P_{3}=P_{2}=\{\tilde{p}\}=\frac{3}{4}$.

\section{2}

In GoS, there are only three nested sets over which preferences are meaningfully defined, namely $\Delta\left(r^{*} \cup C\right), \Delta(M \cup C)$ and $\Delta(R \cup C)$; the corresponding nested sets of subjective probabilities are $P_{4}, P_{3} \subseteq P_{4}$ and $P_{2} \subseteq P_{3}$. But only $P_{4}$ and $P_{3}$ reflect observable preferences.

The nature of preferences among pure or mixed strategies in $G o S$ is discussed at some length in ASR. A significant further comment is related to Proposition 1. Consider the simple two-person, zero-sum game of "matching pennies", where $r \in$ $\{1,2\}, s \in\{1,2\}$, and $h_{r}(s)=1$ for $r=s, h_{r}(s)=-1$ for $r \neq s$. What ultimately matters to each player in this game is that the opponent not be able to "guess" what he himself will play. That is, each player wants his opponent to assign equal probabilities to both of his own strategies. A simple way of achieving that goal is to adopt the mixed strategy $\left(\frac{1}{2}, \frac{1}{2}\right)$. The clear decision by a player to play according to that mixed strategy might be construed as a violation of Proposition 1, because the pure strategies "heads" or "tail" appear discarded in favor of the mixed strategy.

The proper interpretation of Proposition 1 is different. It is simply claimed that a player adopting the mixed strategy $\left(\frac{1}{2}, \frac{1}{2}\right)$ thereby reveals indifference between even- 
tually playing "head" or "tail". Such indifference is consistent with the assignment of equal probabilities to the opponent playing "head" or "tail", and difficult to reconcile with any other assignment. Proposition 1 claims neither more nor less.

In every game situation, a full analysis of the game is needed to form reasonable expectations about the choice(s) of an opponent. We argue here that, under reasonable assumptions, these expectations admit a subjective probability representation, sustaining the retained strategy(ies) as maximising expected utility. The role of game theory in guiding expectations in $G o S$ is then seen as logically equivalent to that of (Bayesian) statistics in GaN.

Open Access This article is distributed under the terms of the Creative Commons Attribution License which permits any use, distribution and reproduction in any medium, provided the original author(s) and source are credited.

\section{References}

Anscombe FJ, Aumann RJ (1963) A definition of subjective probability. Ann Math Stat 34:199-205

Aumann RJ (1962) Utility theory without the completeness axiom. Econometrica 30:445-462

Aumann RJ, Drèze JH (2009) Assessing strategic risk. Am Econ J Microecon 1(1):1-16 (ASR in the text) Castagnoli E, Maccheroni F, Marinacci M (2003) Expected utility with multiple priors. In: Bernard JM, Seidenfeld T, Zaffalon M (eds) Proceedings of the third international symposium on imprecise probabilities and its applications. Carleton Scientific, Waterloo (Canada), pp 121-132

Drèze JH (1987) Decision theory with moral hazard and state-dependent preferences. In: Drèze JH (ed) Essays on economic decisions under uncertaintanty. Cambridge University Press, Cambridge pp 23-89

Dubra J, Maccheroni F, Ok EA (2004) Expected utility theory without the completeness axiom. J Econ Theory 115:118-133

Luce RD, Raiffa H (1957) Games and decisions. Wiley, New York

Nemhauser GL, Wolsey LA (1988) Integer and combinatorial optimisation. Wiley, New York

Rockafellar RT (1970) Convex analysis. Princeton University Press, Princeton

Samuelson PA (1938) A note on the pure theory of consumer's behaviour. Economica NS 5:61-71

Savage LJ (1954) Foundations of statistics. Wiley, New York

von Neumann J, Morgenstern O (1944) Theory of games and economic behaviour. Princeton University Press, Princeton 